\title{
¿HACIA DÓNDE VA LA ECONOMÍA DE LA SALUD?
}

\author{
Autoras: Beatriz G. López-Valcárcel ${ }^{1}$ \\ Departamento de Métodos Cuantitativos en Economía y Gestión \\ Universidad de Las Palmas de Gran Canaria \\ Angelina Lázaro Alquézar² \\ Departamento de Economía Aplicada. Universidad de Zaragoza
}

Recibido: 30/11/2016

Aceptado: 3/12/2016

En este último capítulo del monográfico nos proponemos aventurar algunas posibles líneas de investigación futuras, de avance de la disciplina o ¿hacia dónde va? El envejecimiento de la población, las enfermedades emergentes con la prosperidad, las migraciones a gran escala, la rápida urbanización y la innovación

\footnotetext{
${ }^{1}$ beatriz.lopezvalcarcel@ulpgc.es

²alazaro@unizar.es
} 
tecnológica en medicina y la extensión de la universalización de la cobertura sanitaria probablemente seguirán siendo un desafío para los sistemas sanitarios (Jakovljevic y Ogura, 2016). Estas circunstancias complejas se alzan como impulsores del desarrollo futuro de la Economía de la Salud, que ha de atender los retos de la limitación de los recursos frente a la expansión de las necesidades; la forma de conseguirlo es con mayor pluralismo (Hansen et al., 2015).

El valor académico se mide por raseros distintos que el social. Priman las innovaciones metodológicas y la sofisticación, el uso inteligente y creativo de los datos y los modelos teóricos elegantes, incluso aunque no sean muy aplicables, dados sus supuestos heroicos. El premio Kenneth J. Arrow al mejor artículo de Economía de la Salud que otorga cada año la International Health Economics Association (iHEA) ${ }^{3}$ constituye un buen referente sobre qué tipo de excelencia se reconoce en la profesión. A lo largo de sus 24 ediciones, desde 1993, pueden apreciarse cambios de temática y foco, desde los problemas más tradicionales de riesgo moral, mercados hospitalarios, calidad y competencia entre proveedores en los primeros años, hasta los trabajos más orientados hacia las políticas, a la salud pública y a las medidas del valor de la salud, de los últimos. Algunos temas tradicionales como el aseguramiento persisten (Gaynor y Gertler, 1995; Gruber 1994; Ma y Mcguire 1997; Fang, Keane y Silverman, 2008; Cebul et al., 2011; Group, $2012)^{4}$, pero el enfoque hoy en día se orienta más hacia las políticas.

Ese renovado interés por las políticas se debe en parte a las posibilidades que abren los nuevos métodos de estimación de contrafacturales y a la emergencia de la fiebre de experimentación social, sobre todo en países en desarrollo, propiciados por los organismos internacionales como el Banco Mundial (Khandker, Koolwal y Samad, 2010). El premio de 2005 recayó en un estudio experimental con niños y lombrices en Kenya (Miguel y Kremer, 2004). Ese experimento ha dado lugar a varias publicaciones en revistas top y es citado continuamente como ejemplo en cursos y monografías de economía del desarrollo. La clave del éxito académico del programa PROGRESA de México ha sido su diseño aleatorizado, que ha permitido publicar un considerable número de artículos en revistas científicas indexadas. Asimismo, se busca cada vez más la identificación de los efectos causales con grandes bases de datos observacionales, cuasi experimentales, o con experimentos naturales.

El desarrollo de la Economía del Comportamiento ha tenido gran repercusión en el área de la salud, por su aplicabilidad y porque conecta con las bases conceptuales del núcleo de la disciplina, que al fin y al cabo nació para abordar asimetrías de información, incertidumbre y comportamientos de racionalidad

${ }^{3}$ La lista completa puede consultarse en http://www.healtheconomics.org/page/ArrowAward

${ }^{4}$ Cada uno de estos estudios recibieron el premio Annual Kenneth J. Arrow Award in Health Economics al año siguiente de su publicación. En concreto son los premiados en 1995, 1996, 1998, 2009, 2012 y 2013 respectivamente. 
limitada de los agentes. Es de esperar que sea uno de los grandes temas en los próximos años. El análisis del comportamiento microeconómico de los profesionales, en particular de los médicos, es otro gran tema, que enlaza directamente con incentivos y diseño de contratos. Ya en 1997, un trabajo sobre la práctica de la medicina defensiva (Kessler y Mcclellan, 1996) fue premiado por iHEA. Sin embargo, el cambio de énfasis hacia la salud es evidente. El trabajo premiado en 2015 (Gottlieb y Clemens, 2014) se pregunta si los incentivos financieros a los médicos en Medicare (programa sanitario público de USA dirigido a un colectivo reducido de individuos) afectan a la salud de sus pacientes, y el de 2014 (Kolstad, 2013) indagaba si la publicación de resultados de los cirujanos individuales tiene consecuencias para la salud de los pacientes.

El valor de la vida y de la calidad de vida relacionada con la salud han sido objeto de atención en la primera mitad de la década de los 2000 (Dow, Philipson y Sala-i-Martin, 1999; Becker, Philipson y Soares, 2005; Murphy y Topel, 2006)5 . Hoy en día hay tendencia a una mayor especificidad. Se manifiesta, por ejemplo, en los estudios específicos sobre cáncer u otras enfermedades. La falta de incentivos para investigar a largo plazo en cáncer, por causa de la miopía de la industria farmacéutica y del diseño de la protección de las patentes (plazo fijo desde que se registra la molécula) es el punto de arranque del último premio (2016) recién otorgado por la IHEA (Budish, Roin y Williams, 2015). Los autores concluyen que se paga un altísimo coste de oportunidad social en términos de años de vida ajustados por calidad.

Muchos trabajos recientes de Economía de la Salud explotan datos que permiten hacer inferencia contrafactural, con experimentos naturales o artificiales, para conocer mejor y moldear el complejo comportamiento humano. Ese cambio de énfasis hacia las políticas ha hecho perder interés por los modelos teóricos complejos plagados de supuestos, lo que nos acerca más a otras disciplinas como la Salud Pública, la Epidemiología o la investigación en servicios sanitarios. Las desigualdades en el planeta preocupan. Sin embargo, únicamente una vez se ha premiado, en 2000, un estudio empírico para los países pobres (Dow, Philipson y Sala-i-Martin, 1999). De hecho, la gran mayoría de los trabajos premiados (9 de los 10 últimos) se refieren a Estados Unidos. Esta tendencia al americano-centrismo ya se detectaba en 2012 por Wagstaff y Culyer (2012). La globalización de las publicaciones académicas (hoy en día las dos revistas tradicionales de la disciplina, Journal of Health Economics y Health Economics tienen comités editoriales mixtos europeo-americanos) termina primando las publicaciones americanas. Volviendo a los premios iHEA a la excelencia, sólo 2 de los 24 artículos se han publicado en una revista de Economía de la Salud (específicamente en el Journal of Health

\footnotetext{
${ }^{5}$ Estudios también galardonados con el premio Annual Kenneth J. Arrow Award in Health Economics en el 2000, 2006 y 2007 repectivamente.
} 
Economics). Entre las revistas generales de Economía destaca con diferencia una americana, la American Economic Review (10 de los 24 artículos premiados, 5 de los 7 más recientes). Por otra parte, el hecho de ser calificado de excelente por iHEA no implica que los trabajos tengan gran impacto en términos de citas: 7 de los 12 premios Arrow concedidos en los primeros años de su existencia no aparecen entre los 300 artículos más citados de la disciplina (Wagstaff y Culyer, 2012).

Además de las tendencias apuntadas, otras áreas siguen desarrollándose, con métodos crecientemente sofisticados y grandes bases de datos. El Big Data está llegando a la Economía de la Salud y cada vez hay más aplicaciones relevantes de la disciplina en países no desarrollados.

La Economía de la Salud, disciplina académica implicada en las políticas públicas, será cada vez más pluralista, destacando sus desarrollos en varios frentes (Hansen et al., 2015) entre los que destacan la Economía del comportamiento y experimental, pero también la imbricación con otras disciplinas, en particular con la Epidemiología y la Salud Pública, y la sofisticación de las herramientas cuantitativas.

Pero sobre todo, la nota que mejor puede definir el futuro, es la conexión entre pensamiento y acción, es decir, el carácter utilitarista de la investigación en Economía de la Salud, en la que un valor añadido de la mima es la capacidad para mejorar el mundo.

Y que el filón de la Economía de la Salud es inagotable.

\section{BIBLIOGRAFÍA}

BECKER, G. S., PHILIPSON, T. J. y SOARES, R. R. (2005), "The quantity and quality of life and the evolution of world inequality", American Economic Review, núm. 95(1), pp. 277-291.

BUDISH, E., ROIN, B. N. y WILLIAMS, H. (2015), "Do firms underinvest in long-term research? Evidence from cancer clinical trials", American Economic Review, núm. 105(7), pp. 2044-2085.

CEBUL, R. D. y otros (2011), "Unhealthy insurance markets: Search frictions and the cost and quality of health insurance", American Economic Review, núm. 101(5), pp. 1842-1871.

DOW, W. H., PHILIPSON, T. J. y SALA-I-MARTIN, X. (1999), "Longevity complementarities under competing risks", American Economic Review, núm. 89(5), pp. 1358-1371.

FANG, H., KEANE, M. P. y SILVERMAN, D. (2008), "Sources of Advantageous Selection: Evidence from the Medigap Insurance Market”, Journal of Political Economy, núm. 116(2), pp. 303-350. 
GAYNOR, M. y GERTLER, P. (1995), "Moral hazard and risk spreading in partnerships", The RAND Journal of Economics, núm. 26(4), pp. 591-613.

GOTTLIEB, J. D. y CLEMENS, J. (2014), "Do Physicians' Financial Incentives Affect Treatment Patterns and Patient Health?", American Economic Review, núm. 2014(4), pp. 1320-1349.

GROUP, O. H. S. (2012), "The Oregon health insurance experiment: evidence from the first year", Quarterly Journal of Economics, núm. 127(3), 1057-1106,

GRUBER, J. (1994), "The incidence of mandated maternity benefits", American Economic Review, núm. 84(3), pp. 622-641.

HANSEN, F. et al. (2015), "The future of health economics: the potential of behavioral and experimental economics", Nordic Journal of Health Economics, núm. 3(1), pp. 68-86.

JAKOVLJEVIC, M. M. y OGURA, S. (2016), "Health Economics at the Crossroads of Centuries - From the Past to the Future", Frontiers in Public Health, núm. 4, p. 115.

KESSLER, D. y MCCLELLAN, M. (1996), "Do Doctors Practice Defensive Medicine?", The Quarterly Journal of Economics, 111(2), pp. 353-390.

KHANDKER, S. R., KOOLWAL, G. B. y SAMAD, H. A. (2010), Handbook on impact evaluation: quantitative methods and practices, World Bank Publications.

KOLSTAD, J. T. (2013), "Information and quality when motivation is intrinsic: Evidence from surgeon report cards", The American Economic Review, núm. 103(7), pp. 2875-2910.

MA, C.-T. A. y MCGUIRE, T. G. (1997), "Optimal health insurance and provider payment", The American Economic Review, núm. 87(4), pp. 685704.

MIGUEL, E. y KREMER, M. (2004), "Worms: identifying impacts on education and health in the presence of treatment externalities", Econometrica, núm. 72(1), pp. 159-217.

MURPHY, K. M. y TOPEL, R. H. (2006), "The Value of Health and Longevity", Journal of Political Economy, 114(5).

WAGSTAFF, A. y CULYER, A. J. (2012), "Four decades of health economics through a bibliometric lens", Journal of Health Economics, núm. 31(2), pp. 406-439. 